\title{
Systematic study of bimodal suspensions of latex nanoparticles using dynamic light scattering
}

\author{
A. K. Jamting ${ }^{1}$, J. Cullen ${ }^{1,2}$, V. A. Coleman ${ }^{1}$, M. Lawn ${ }^{1}$, J. Herrmann ${ }^{1}$, J. Miles ${ }^{1}$ and \\ M. J. Ford ${ }^{2}$ \\ ${ }^{1}$ Nanometrology Section, National Measurement Institute, PO Box 264, Lindfield, \\ NSW 2070, Australia \\ ${ }^{2}$ Institute for Nanoscale Technology, University of Technology Sydney, PO Box 123, \\ Broadway, NSW 2007, Australia
}

e-mail of corresponding author: Asa.Jamting@measurement.gov.au

\begin{abstract}
Determining the size of nanoparticles accurately, quickly and easily is becoming more and more important as the use of such particles increases. One of the common techniques for measuring the size of particles in suspension is dynamic light scattering (DLS). In principle, DLS is able to estimate the average particle size and its distribution. However, the measured correlation function or power spectrum must be inverted to obtain this size distribution. The inversion is an ill-conditioned mathematical problem, and only under certain assumptions can the distribution be determined reliably. Suspensions containing bimodal (or multi-modal) particle size distributions are particularly challenging. This study reports on DLS measurements on a range of bimodal distributions of latex spheres with varying fractions of particle sizes. To determine the efficacy of different inversion techniques, the data has been analysed both with the algorithms implemented in the instrument's proprietary analysis software and with other inversion routines based on simple analytical models of the particle size distribution.. In addition, the results of the DLS analysis have been compared to scanning and transmission electron microscopy (SEM and TEM) and atomic force microscopy (AFM) measurements.
\end{abstract}

\section{Introduction}

There is growing interest in the ability to accurately determine the size of nanoparticles quickly and easily, as the use of such particles increases in a range of applications. One of the common techniques for measuring the size of small particles in suspension is dynamic light scattering (DLS). The technique is based upon measurement of a time-dependent fluctuating light intensity signal scattered by particles in a suspension that undergo Brownian motion. The autocorrelation function of the scatter intensity for monodisperse particles decays exponentially with a decay rate that is proportional to the diffusion coefficient $D$ of the particle motion which, in turn, is related to the hydrodynamic particle diameter $\mathrm{d}$ via the Stokes-Einstein relation [1]. For polydisperse systems, the expression for the autocorrelation function (ACF) becomes an integral equation which must be inverted to obtain the particle size distribution (PSD). The inversion presents a mathematical problem which is ill-conditioned, and only under certain assumptions can the PSD be determined reliably.

The theoretical considerations used to obtain the particle size have been presented in great detail elsewhere [2],[3]. The method for determining the diffusion coefficient, and hence the particle size, is based on the cumulants method [4]. A range of different data analysis methods have been developed based on nonnegatively constrained least-squares (NNLS) models, such as for example CONTIN [5]. The analysis of polydisperse systems, however, remains problematic. Most commercial DLS instruments on the market today are optimized for ease of use, with little or no user access to the analysis parameters. This approach works very well for a large number of applications, but may often fail to accurately determine the size of nanoparticles, and particularly nanoparticles in bimodal or multi-modal systems which represent more realistic suspensions. This study, therefore, focuses on well-defined bimodal suspensions of nanoparticles. The particle systems under investigation consist of certified reference materials (CRMs) of polystyrene latex (PSL) spheres. Different approaches to the analysis of DLS 
data for these systems have been trialed. The first approach was to analyze the data using the various analysis models available in the proprietary software of the DLS instrument. Subsequently, alternative mathematical algorithms were tried in an effort to improve the analysis. In addition, the influence of correlator settings has been investigated both theoretically and experimentally to explore the possibility of further improving the accuracy of the measurement of the bimodal PSDs.

\section{Experimental procedure}

In this study, the samples were measured in a Malvern Zetasizer Nano DLS system [6] fitted with the Research version of the software. All the samples used in the current study consist of certified PSL spheres from Thermo Scientific (formerly Duke Scientific) [7] with sizes varying from $20 \mathrm{~nm}$ to $100 \mathrm{~nm}$. The concentrated suspensions of PSL particles were diluted with $10 \mathrm{mmol}$ sodium chloride in ultrapure water (resistivity $18.2 \mathrm{M} \Omega \mathrm{cm}$ ) [8]). The samples comprise of either monomodal suspensions of each particle size or specific bimodal suspensions of two defined particle sizes. The bimodal samples were prepared by mixing $100 \mathrm{~nm}$ particles with known volume quantities of smaller particles $(20 \mathrm{~nm}, 30 \mathrm{~nm}, 40 \mathrm{~nm}$, $50 \mathrm{~nm}, 60 \mathrm{~nm}, 70 \mathrm{~nm}, 80 \mathrm{~nm}$ and $90 \mathrm{~nm}$ ). The total concentration of particles in the suspension was $\sim 5 \%$. Subsequently, microscopic analysis to verify the DLS results was carried out using a Philips CM12 TEM and a Zeiss Supra SEM.

\section{Results and discussion}

\section{DLS}

In order to investigate the best conditions for differentiating between the two different sizes in the bimodal suspensions, a range of volume ratios were trialed. Typical results from experiments on samples containing $20 \mathrm{~nm}$ and $100 \mathrm{~nm}$ particles at volume ratios of 20:1, 20:2 and 20:3 are shown in Figure 1 below. These results show a greater resolving power for the 20:1 ratio, and the results which follow are from experiments using this ratio.

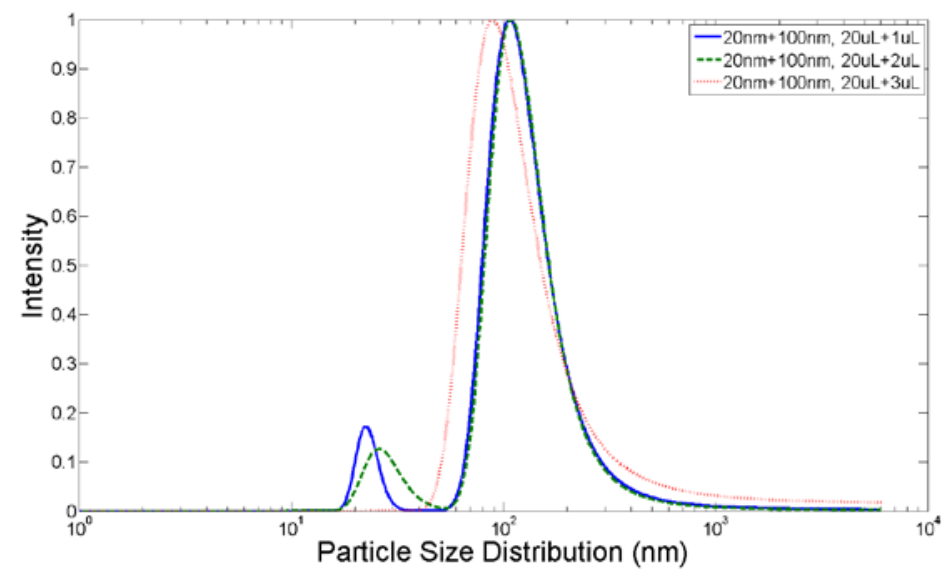

Figure 1. The results from DLS measurements on bimodal suspensions of $20 \mathrm{~nm}$ and $100 \mathrm{~nm}$ PSL spheres with three different volume ratios (20:1, 20:2 and 20:3), analysed using proprietary software.

The experimental results were first analysed using the proprietary software. There are two models available for sample analysis, a general purpose model and a model optimised for samples with multiple narrow modes (MNM). 

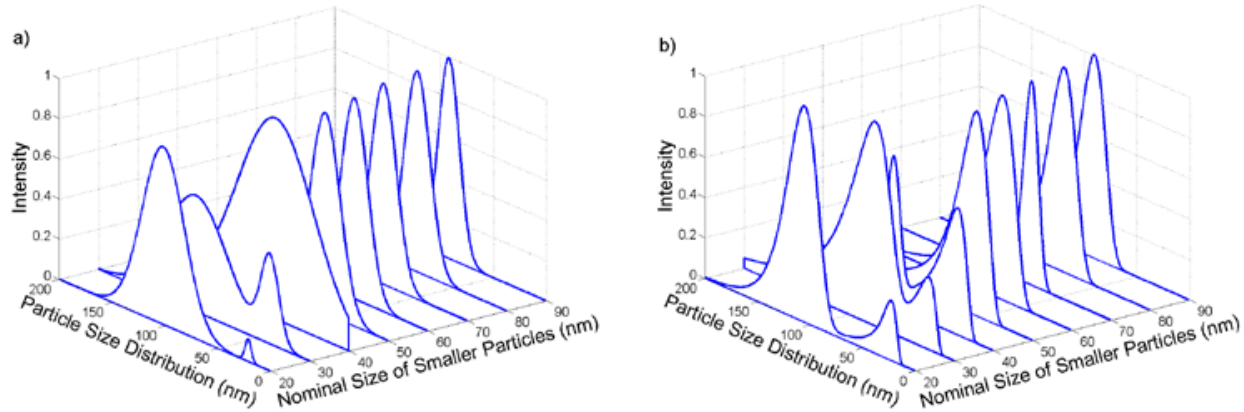

Figure 2. Analysis of DLS measurements of 8 different bimodal suspensions. a) shows the results when analysed using proprietary 'multiple narrow mode' software. The figure shows that the bimodal peaks are only resolved for the samples containing $20+100 \mathrm{~nm}$ and $30+100 \mathrm{~nm}$ particles. b) shows the results when the same dataset is analysed using an alternative analytical model (see text for details). The figure shows that the bimodal peaks are now also resolved for the sample containing $40+100 \mathrm{~nm}$ particles.

The general model was unable to resolve the individual peaks in the PSDs for the bimodal samples while the MNM model resolved two peaks for the samples containing $20+100 \mathrm{~nm}$ particle and $30+100 \mathrm{~nm}$ particles. Beyond that ratio, the software only found one broad peak, as shown in the graph in Figure 2 a).

The analysis of the same DLS data was then repeated using alternative analysis methods that model the autocorrelation function in terms of a weighted distribution of decay rates. The model uses a distribution which comprises individual peaks resulting from a Taylor expansion around a mean decay rate up to second order [9]. This alternative analysis improved the resolution of the bimodal PSDs as illustrated in Figure 2 b).

To assess the influence of the correlator settings, synthetic ACF data was generated. The number of ACF data points, i.e. their delay time spacing, affects the results strongly. For higher numbers of ACF data points, the analysis was able to resolve separate peaks for bimodal samples up to $80+100 \mathrm{~nm}$, as shown in Figure 3. However, in order to obtain this high level of resolution, the number of ACF data points had to be increased from 192 (representing the correlator of the DLS instrument) to 500. Figure 3 illustrates the improved resolving power resulting from that increase of ACF data points.
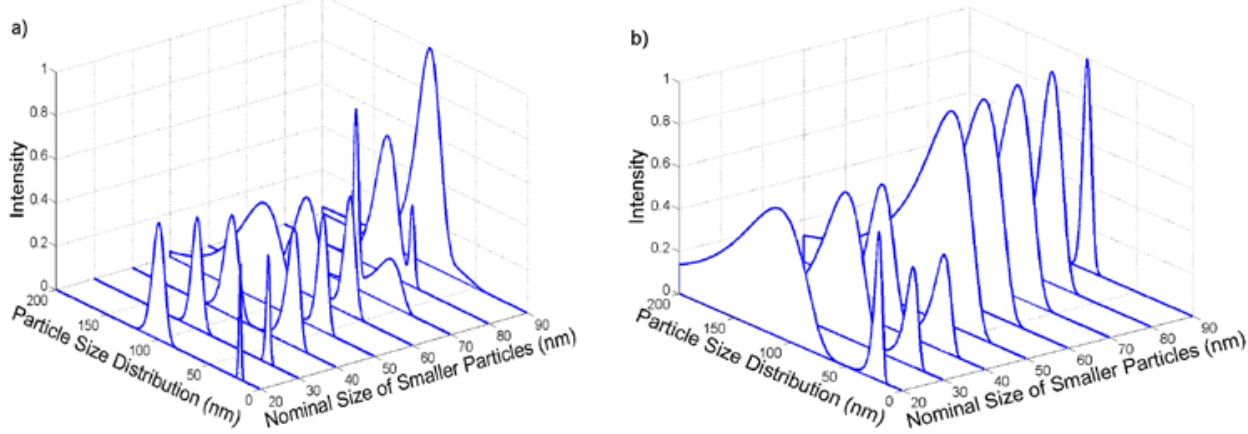

Figure 3. Analysis of synthetic ACF data corresponding to bimodal suspensions containing mixtures of $100 \mathrm{~nm}$ particles and particles ranging in size from $20 \mathrm{~nm}$ to $90 \mathrm{~nm}$. a) shows the results for ACF data based on 500 data points and b) shows the results obtained using the correlator settings of the DLS instrument used in this study (192 ACF data points). 


\section{Microscopy}

To verify the accuracy of the DLS analysis, the results were compared to SEM and TEM and AFM measurements. Some typical micrographs are shown in Figure 4. The particle sizes obtained from microscopy are consistent with the DLS results. TEM (as well as SEM) images of the $100 \mathrm{~nm}$ PSL samples show a narrow PSD. The width of the PSD for the smaller diameter spheres seems to be larger, as evident in Figure $4 \mathrm{~b}$ ), which shows an SEM image of the $50+100 \mathrm{~nm}$ sample. The volume ratio of $50 \mathrm{~nm}$ to $100 \mathrm{~nm}$ particles (20:1, typical for this study in order to generate two distinguishable peaks in the DLS measurements) is well illustrated here, with only six $100 \mathrm{~nm}$ particles present among the large number of nominally $50 \mathrm{~nm}$ particles.
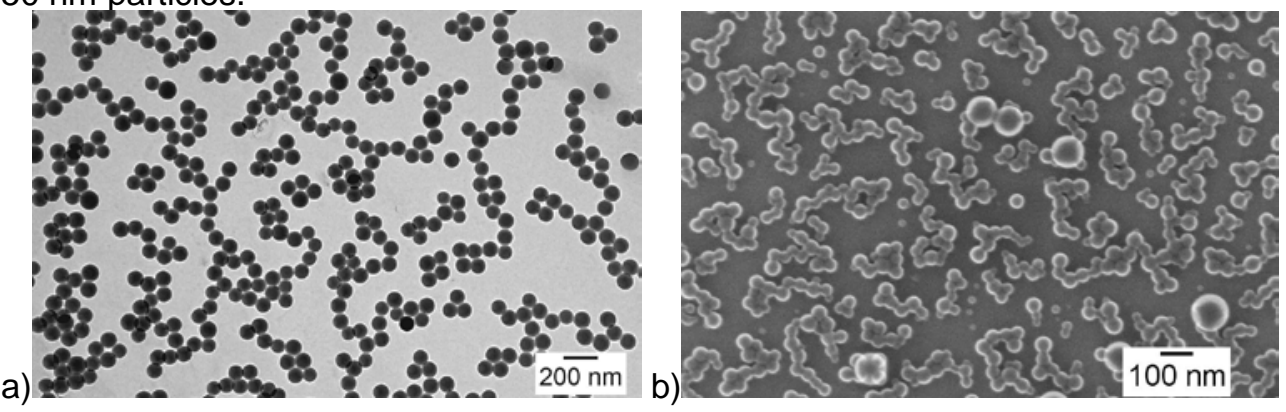

Figure $4 . \quad$ a) TEM image of monomodal $100 \mathrm{~nm}$ PSL particles and b) SEM image of the $50 \mathrm{~nm}+100 \mathrm{~nm}$ bimodal sample.

\section{Summary and Conclusions}

In this study, the ability to accurately determine the size of bimodal distributions of nanoparticles using DLS has been investigated. The theoretical as well as experimental results from the study indicate that by carefully choosing the mathematical model used in the analysis of the experimental data and by maximizing the number of data points in the ACF it is possible to improve the resolving power of the analysis for bimodal nanoparticle suspensions. Knowledge of the particle system under investigation is vital for the analysis, and future work will include further studies of the effect of correlator settings as well as investigation of different types of nanoparticles and of systems with unknown sizes.

\section{Acknowledgements}

The authors wish to acknowledge the Microstructure Analysis Unit at the University of Technology, Sydney for access to the scanning electron microscope and the Electron Microscopy Unit at the University of Sydney for access to the transmission electron microscope.

\section{References}

[1] R. Finsy, Particle Sizing by Quasi-Elastic Light Scattering, Advances in Colloid and Interface Science, 52, 79-143, 1994

[2] B.J. Berne and R. Pecora, 2000, Dynamic light scattering: with applications to chemistry, biology and physics, Dover Press, New York,

[3] B. Chu and T. Liu, Characterization of nanoparticles by scattering techniques, Journal of Nanoparticle Research 2, 29-41, 2000

[4] ISO 13321 Particle Size Analysis - Photon Correlation Spectroscopy, International Organization for Standardization, Geneva (1996)

[5] S. Provencher, CONTIN: A general purpose constrained regularization program for inverting noisy linear algebraic and integral equations, Computer Physics Communications, 27, 229-242, 1982

[6] Malvern Instruments Ltd, UK.

[7] Thermo Scientific Corporation, USA.

[8] Millipore Milli-Q Gradient system, Millipore Australia Pty Ltd.

[9] B. Frisken, Revisiting the method of cumulants for the analysis of dynamic light-scattering data, Applied Optics, 40, 4087-4091, 2001 